\title{
Aiding the Design of Radiation Resistant Materials with Multiphysics Simulations of Damage Processes
}

C. P. Race ${ }^{1}$, D. R. Mason ${ }^{1}$, J. le Page ${ }^{1}$, M. W. Finnis ${ }^{1,2}$, W. M. C. Foulkes ${ }^{1}$ and A. P. Sutton ${ }^{1}$

${ }^{1}$ Department of Physics, Imperial College London, London, SW7 2AZ, United Kingdom.

${ }^{2}$ Department of Materials, Imperial College London, London, SW7 2AZ, United Kingdom.

\section{ABSTRACT}

The design of metals and alloys resistant to radiation damage involves the physics of electronic excitations and the creation of defects and microstructure. During irradiation damage of metals by high energy particles, energy is exchanged between ions and electrons. Such "nonadiabatic" processes violate the Born-Oppenheimer approximation, on which all conservative classical interatomic potentials rest. By treating the electrons of a metal explicitly and quantum mechanically we are able to explore the influence of electronic excitations on the ionic motion during irradiation damage.

Simple theories suggest that moving ions should feel a damping force proportional to their velocity and directly opposed to it. In contrast, our simulations of a forced oscillating ion have revealed the full complexity of this force: in reality it is anisotropic and dependent on the ion velocity and local atomic environment. A large set of collision cascade simulations has allowed us to explore the form of the damping force further. We have a means of testing various schemes in the literature for incorporating such a force within molecular dynamics (MD) against our semi-classical evolution with explicitly modelled electrons. We find that a model in which the damping force is dependent upon the local electron density is superior to a simple fixed damping model. We also find that applying a lower kinetic energy cut-off for the damping force results in a worse model. A detailed examination of the nature of the forces reveals that there is much scope for further improving the electronic force models within MD.

\section{INTRODUCTION}

A radiation damage cascade begins when an ion in a solid is set in motion by collisions with incoming particulate radiation or the products of radioactive decay processes. This primary knock-on atom (PKA) then goes on to collide with other atoms of the solid creating damage to the material at the atomic level. The initial disruption occurs rapidly on a picosecond timescale and over a region hundreds of nanometers in size. Following the point of maximum damage there is a period of hundreds of ps, during which many of the defects recombine and the lattice largely heals itself to leave a residual defect distribution that forms the initial conditions for the long-term micro-structural evolution of the material [1].

As radiation damage takes place on length and timescales at or beyond the resolution of experimental investigation, much of what we know about cascade evolution has been established via simulation. Classical molecular dynamics (MD) simulations can now handle sufficiently large systems to allow the direct simulation of high energy collision cascades [2]. However, it is well established [3] that the swift motion of ions during a collision cascade can excite the electrons out of their ground state. The cumulative effect of many small electronic excitations is to raise the electronic temperature, away from equilibrium with the ionic temperature [17]. This provides a mechanism of ionic energy loss - if the electronic thermal conductivity is high then energy is rapidly transferred out of the cascade region. These effects are ignored by standard classical MD simulations.

Several augmented MD schemes [4-6] have attempted to model the effect of electrons on 
ionic motion by including extra forces. Stopping power theories such as the Lindhard's dielectric theory [7] of a uniform electron gas and binary collision treatments by Firsov [8] and Lindhard and Scharff $[9,10]$ suggest that for ions with velocities up to around $10 \AA \mathrm{fs}^{-1}$ the electrons provide a drag force, proportional to the ion velocity and directly opposed to it, $\mathbf{F}=-\beta \mathbf{v}$.

In our work we have the physics necessary to accurately capture energy transfer from ions to electrons in radiation damage in metals. Each ion in a collision cascade interacts with many others in a complex, changing environment, so we use a model that allows for the evolution of explicitly quantum mechanical electrons coupled to the motion of classical ions. By choosing a simple model we can directly simulate collision cascades, and so explore the effect of irreversible energy transfer into the electronic subsystem on the ion dynamics in radiation damage events. This model thus provides a means of testing the validity of applying simple drag forces in classical MD.

\section{THEORY AND SIMULATION}

Direct simulation of a set of quantum mechanical ions and electrons is computationally intractable, but the semi-classical approximation, in which the electrons are treated quantum mechanically and ions are modeled as classical particles, offers a justifiable compromise. We adopt the Ehrenfest approach, which accurately reproduces the true electron-ion interaction when the temperature of the ionic subsystem is much greater than that of the electronic subsystem [11]. Within this approximation the ions move under 'mean-field' forces due to the electrons and the electrons are evolved quantum mechanically under a Hamiltonian $\hat{H}(\mathbf{R}(t))$ parameterized by the nuclear trajectories in position space $\mathbf{R}(t)=\left\{\mathbf{R}_{1}(t), \mathbf{R}_{2}(t), \ldots, \mathbf{R}_{N}(t)\right\}$. We find that self-consistent electron-electron interactions make little difference to the results, so use a non-self-consistent Hamiltonian. The electrons are represented by a single particle density matrix $\hat{\rho}(t)$ evolving according to the quantum Liouville equation, the density matrix analog of the time-dependent Schrödinger equation:

$$
\mathrm{i} \hbar \frac{\partial \hat{\rho}}{\partial t}=[\hat{H}(\mathbf{R}(t)), \hat{\rho}(t)] \text {. }
$$

The ions evolve under the equation

$$
M_{n} \ddot{\mathbf{R}}_{n}(t)=-\nabla_{\mathbf{R}_{n}} V_{\text {ion }}(\mathbf{R}(t))-2 \operatorname{Tr}\left\{\hat{\rho}(t) \nabla_{\mathbf{R}_{n}} \hat{H}(\mathbf{R}(t))\right\},
$$

where ion $n$ has mass $M_{n}$ and the 2 is for spin degeneracy. $V_{\text {ion }}(\mathbf{R}(t))$ includes repulsive ion-ion interactions and the second term is a Hellman-Feynman force due to the electrons.

We adopt the single $s$-band orthogonal tight-binding model of Sutton et al. [12], in which the hopping integrals between atom-centred orbitals are parameterized as an inverse power of the inter-ionic separation. This model gives us a metallic electronic structure and the structural and elastic properties of copper, and is simple enough to allow us to reach the required system sizes.

Because we explicitly evolve the electronic subsystem, we have access, via the density matrix $\hat{\rho}(t)$ to all its properties. Here we focus on the irreversible energy transfer from the ionic system into the electrons, which we denote $\Delta E_{\mathrm{Ehr}}(t)$. We define this quantity as the difference between the energy in the electronic system at time $t$,

$$
E(t)=2 \operatorname{Tr}\{\hat{\rho}(t) \hat{H}(\mathbf{R}(t))\},
$$

and the energy that the electrons would have had if the ions had traversed their trajectories infinitely slowly, the adiabatic energy $E_{\mathrm{Ad}}(\mathbf{R}(t))$. We can calculate this energy by noting that in the adiabatic limit the $n^{\text {th }}$ eigenstate $\left|\psi_{n}\left(t_{0}\right)\right\rangle$ of the initial Hamiltonian $\hat{H}\left(\mathbf{R}\left(t_{0}\right)\right)$ will evolve 
exactly into the corresponding (in an energy ordered list) eigenstate $\left|\psi_{n}(t)\right\rangle$ of $\hat{H}(\mathbf{R}(t))$ at some later time [16].

Our studies of the irreversible energy transfer due to a single oscillating ion [13] suggest that the effective damping constant of a moving ion is a tensor function of its speed, direction, local atomic environment and electronic temperature. We are thus motivated to apply our simulation technique to investigate the validity of various classical MD models for the electronic damping of ionic motion prevalent in the literature.

We have carried out a series of 240 cascade simulations in 2016 atom supercells $(9 \times 8 \times 7$ fcc unit cells). In each simulation a single ion in a perfect static lattice is given an initial kinetic energy in a particular direction. Our set of simulations takes ten energies evenly distributed between $100 \mathrm{eV}$ and $1 \mathrm{keV}$ and directs the ion in each case in twenty-four different directions evenly distributed over the irreducible $1 / 48^{\text {th }}$ of the fcc cell. For numerical convenience the density matrix is initialized with a small, finite electronic temperature $T_{e}=500 \mathrm{~K}$. Each resulting cascade is evolved for $200 \mathrm{fs}$ and we record the irreversible energy transfer $\Delta E_{\mathrm{Ehr}}(t)$ and the ionic trajectories $\mathbf{R}(t)$. For any given classical damping model we can now calculate a damping constant $\beta_{n}(t)$ for each atom at each time and calculate the work that would have been done by the corresponding classical drag forces as the ions traversed the trajectories derived from our quantum mechanical cascade simulations,

$$
\Delta E_{\text {Model }}(t)=\int_{0}^{t} \mathrm{~d} t^{\prime} \beta_{n}\left(t^{\prime}\right) \mathbf{R}\left(t^{\prime}\right) \cdot \mathbf{R}\left(t^{\prime}\right) .
$$

We would like for there to exist some classical conservative potential, which when supplemented by a given drag force model reproduces exactly the ion trajectories. Should we find that no parameterization of $\beta_{n}(\mathbf{R}(t))$ gives $\Delta E_{\mathrm{Ehr}}(t)=\Delta E_{\text {Model }}(t)$, then no such classical potential can exist. We may then conclude that the assumptions underlying the drag model are faulty. The models that we choose to test are:

1. A simple damping constant applied to all ions at all velocities, $\beta_{n}(t)=\beta$. This is the $T_{\mathrm{e}}=0$ limit of the model due to Finnis et al. [4], applied by, for example, Ref. [14].

2. A simple damping coefficient, but applied only to ions with a kinetic energy greater than $10 \mathrm{eV}: \beta_{n}(t)=\beta$ for $\frac{1}{2} M v_{n}{ }^{2} \geq 10 \mathrm{eV}, \beta_{n}(t)=0$ for $\frac{1}{2} M v_{n}{ }^{2}<10 \mathrm{eV}$. This scheme has been implemented by Nordlund et al. [5]. While no thorough justification for this form is offered in the literature, the cutoff is generally understood to ensure the damping does not cool the ions to $0 \mathrm{~K}$.

3. A model in which the damping is a function of the local electron density $\rho(\mathbf{r}, t)$, made use of by embedded atom potentials: $\beta_{n}(t)=\beta\left[\rho\left(\mathbf{R}_{n}, t\right)\right]$. A model of this form was proposed by Caro and Victoria [6] and implemented in Ref. [15].

\section{RESULTS AND DISCUSSION}

In figure (1) we compare the irreversible energy transfer from our simulations with the value calculated in each of the three classical models under test. Models 1 and 3 (the simple damping and density-dependent damping respectively) appear to do a good job of capturing the energy transfer throughout the simulations. Model 2, in contrast, performs much worse, failing to capture the energy transfer from slower moving ions (below the kinetic energy cut-off) later in the cascade. 

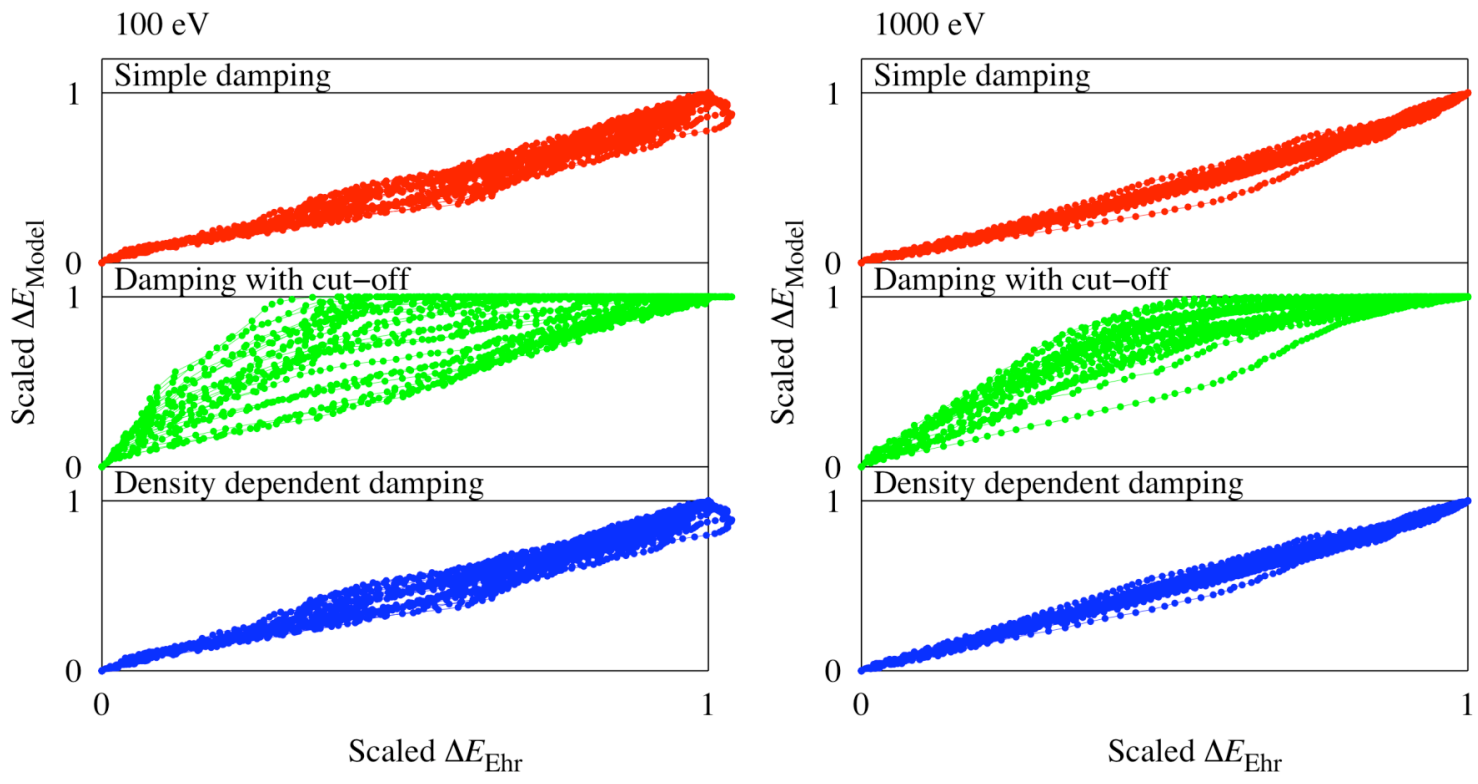

Figure 1. Scatter plots of the irreversible energy transfer calculated by three different classical models against the energy transfer found in simulations with Ehrenfest dynamics. The left hand panel shows data for all 24 initial PKA directions for a kinetic energy of $100 \mathrm{eV}$. The right hand panel shows the same for initial kinetic energy $1000 \mathrm{eV}$. The data are scaled by the energy transfer found by each method at $200 \mathrm{fs}$, so that a perfect match between a classical method and the Ehrenfest results would appear as a straight line of gradient 1. Models 1 (simple damping top panels) and 3 (density-dependent damping - bottom panels) are closest to linear, indicating that they best reproduce the Ehrenfest energy transfer. Model 2 (damping with cut-off - middle panels) fails to capture the energy loss from slow moving ions.

For each simulation and for each model, we now compare $\Delta E_{\mathrm{Ehr}}(t)$ and $\Delta E_{\mathrm{Model}}(t)$ at intervals of $2.5 \mathrm{fs}$ throughout the simulation. Using these data, we calculate a best-fit value for the parameters of the models using a least-squares fitting algorithm. The average best-fit values calculated for the damping constant $\bar{\beta}$ are shown in figure (2). In the figure we have separated out those simulations in which the PKA was given an initial velocity along the $\langle 110\rangle$ closepacked direction. In these cases much of the cascade energy is confined to a single close-packed row of ions within what is known as a replacement collision sequence (RCS). As can be seen from the simple damping model (model 1) the damping of such a mode of ionic motion is significantly enhanced over that experienced in a general cascade. Model 2 ignores the damping of slow moving ions, so requires a higher average damping constant in order to account for the energy loss. The damping coefficients for model 3 are much lower than those for the other models. The values of $\bar{\beta}$ reported for model 3 in the figure correspond to the damping experienced by an atom at a perfect lattice site. In a cascade the moving atoms will generally experience higher electron densities, and so the damping experienced by the ions will be higher than that suggested by a simple reading of figure (2a). For all models, the computed damping coefficient is insensitive to the PKA energy, lending support to the idea of a velocity independent damping coefficient. 

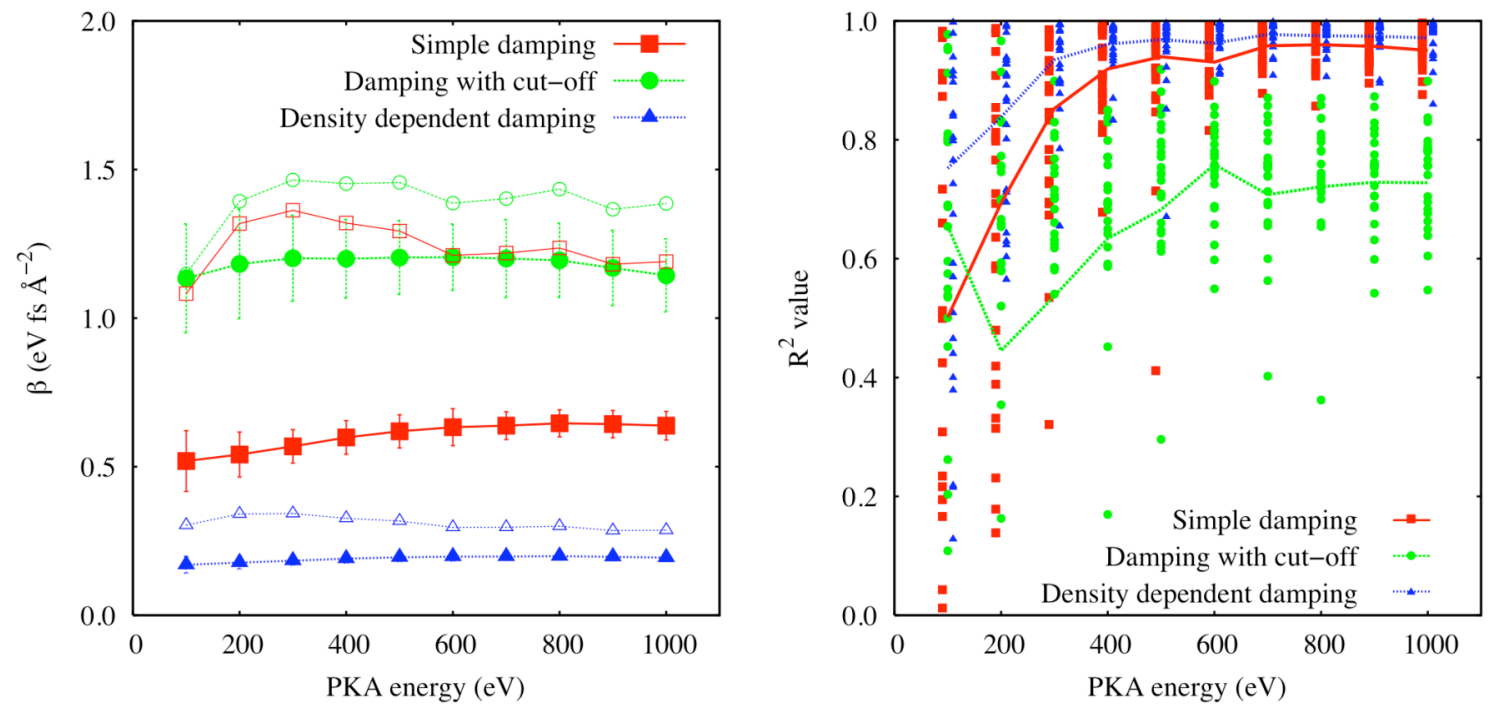

Figure 2. Left panel: The damping coefficient as a function of PKA energy calculated for each of the three classical models under test. The larger solid symbols are an average across all PKA directions except for the $\langle 110\rangle$ direction. Best-fit damping coefficients for the simulations in the $\langle 110\rangle$ direction are shown by smaller open symbols. Lines are included as a guide for the eye.

Right panel: $R^{2}$ measures of goodness of fit for the three classical models under test. Each data point represents one cascade simulation. The lines join the averages of the $R^{2}$ values for each model at each PKA energy (simulations in the $\langle 110\rangle$ directions are excluded) and provide a guide for the eye.

From figure (2a) we can extract a single value for $\bar{\beta}$ computed from the higher energy cascades, and use this to generate $R^{2}$ goodness of fit statistics for the least-squares fitting of the damping coefficients in all cascades (Figure $2 \mathrm{~b}$ ). These results support the representation of the electrons simply by adding a viscous damping (model 1). Even better is to make the viscosity environmentally dependent (model 3). There is little support for using a kinetic energy cut-off (model 2) .

\section{CONCLUSIONS}

We have developed a semi-classical simulation scheme for use in simulating radiation damage processes in metals. By incorporating quantum mechanical electrons coupled to classical ions we can directly explore the effect of the irreversible transfer of energy from ions to electrons. By keeping the electronic model as simple as possible we are able to reach the length and time-scales necessary to directly simulate radiation damage phenomena, which as yet remain beyond the reach of time-dependent density functional theory. However this does imply that the method should be understood as a means of exploring the relevant physics rather than being able to make chemically specific predictions.

In the present work we test the validity of three commonly used models for the damping effect of electrons on ionic motion. We have found that, on average across the evolution of small collision cascades, simple damping models can do a remarkably good job of capturing the electronic damping effect. In particular, we have found that a local environment dependence (via the local electron density) proposed by Caro and Victoria [6] significantly enhances the 
correspondence between the classical and quantum mechanical calculations of irreversible energy transfer. However, we find no justification for the use of a lower kinetic energy cut-off for the application of the damping. Such a cut-off makes the correspondence with the quantum mechanical energy transfer significantly worse.

\section{ACKNOWLEDGMENTS}

We thank IC-HPC for computing resources. CRP and DRM were supported by EPSRC grant number EP/C524403.

\section{REFERENCES}

1. R. S. Averback, and T. Diaz de la Rubia, Solid State Phys. 51, 281-402 (1997).

2. L. Malerba, J. Nucl. Mater. 351, 28-38 (2006).

3. P. Sigmund. Particle penetration and radiation effects - General aspects and stopping of swift point charges. Springer-Verlag Berlin / Heidelberg (2006).

4. M. W. Finnis, P. Agnew, and A. J. E. Foreman. Phys. Rev. B, 44, 567-74 (1991)

5. K. Nordlund, M. Ghaly, and R. S. Averback. J. Appl. Phys., 83,1238-46 (1998).

6. A. Caro and M. Victoria. Phys. Rev. A, 40, 2287-91 (1989).

7. J. Lindhard. Mat Fys Medd Dan Vid, 8, 28 (1954).

8. O. B. Firsov. Soviet Physics JETP, 36, 1076 (1959) .

9. J. Lindhard and M. Scharff. Phys. Rev. 124,128-30 (1961) .

10. I. S. Tilinin. Phys. Rev. A, 51, 3058-65 (1995).

11. J. le Page, D. R. Mason, and W. M. C. Foulkes. J. Phys-Condens. Mat., 20, 125212 (2008).

12. A. P. Sutton, T. N. Todorov, M. J. Cawkwell, and J. Hoekstra. Phil.Mag.A, 81,1833 (2001).

13. D. R. Mason, J. le Page, C. P. Race, W. M. C. Foulkes, M. W. Finnis, and A. P. Sutton. J. Phys-Condens. Mat., 19, 436209 (2007).

14. F. Gao, D. J. Bacon, P. E. J. Flewitt, and T. A. Lewis. Model Simul Mater SC, 6, 543 (1998). 15. A. Duvenbeck and A. Wucher. Physical Review, 72, 165408 (2005).

16. J. le Page, D. R. Mason, C. P. Race, and W. M. C. Foulkes. New J. Phys, 11, 013004 (2009) 17. C. P. Race, D. R. Mason, and A. P. Sutton. J. Phys-Condens. Mat., 21, 115702 (2009). 